OPEN ACCESS

Edited by:

David R. Mandel,

Defence Research and Development

Canada, Canada

Reviewed by:

Jan Sprenger,

Tilburg University, Netherlands

Peter Baumann,

Swarthmore College, USA

*Correspondence:

Jean Baratgin,

Laboratory CHArt, Université Paris 8, Site Paris-EPHE: 4-14 rue Ferrus,

75014 Paris, France

jean.baratgin@univ-paris8.fr

Specialty section:

This article was submitted to

Cognition,

a section of the journal

Frontiers in Psychology

Received: 30 March 2015

Accepted: 24 July 2015

Published: 11 August 2015

Citation:

Baratgin J (2015) Rationality, the Bayesian standpoint, and the

Monty-Hall problem.

Front. Psychol. 6:1168.

doi: 10.3389/fpsyg.2015.01168

\section{Rationality, the Bayesian standpoint, and the Monty-Hall problem}

\author{
Jean Baratgin ${ }^{1,2 *}$ \\ ${ }^{1}$ Laboratory CHArt (PARIS), Université Paris 8, Paris, France, ${ }^{2}$ Institut Jean Nicod, Paris, France
}

The Monty-Hall Problem (MHP) has been used to argue against a subjectivist view of Bayesianism in two ways. First, psychologists have used it to illustrate that people do not revise their degrees of belief in line with experimenters' application of Bayes' rule. Second, philosophers view MHP and its two-player extension $\left(\mathrm{MHP}_{2}\right)$ as evidence that probabilities cannot be applied to single cases. Both arguments neglect the Bayesian standpoint, which requires that $M H P_{2}$ (studied here) be described in different terms than usually applied and that the initial set of possibilities be stable (i.e., a focusing situation). This article corrects these errors and reasserts the Bayesian standpoint; namely, that the subjective probability of an event is always conditional on a belief reviser's specific current state of knowledge.

Keywords: Bayesian standpoint, Monty-Hall problem with two players, probability revision, collider principle, single case probability

\section{Introduction}

In the Monty Hall Problem (MHP), you know that the car you want is behind one of three closed doors and a goat behind the other two doors. You choose a door and Monty (the host who knows where the car is) opens another door with a goat behind (as you know he can neither open your door nor a door with the car behind). After the host's action, would you rather stick to your original choice or switch to the remaining door?

MHP is a much-studied experimental paradigm investigating the inability of (naive and expert) people to revise their degrees of belief in a Bayesian manner (for a recent review see Tubau et al., 2015). Specific reformulations of format (natural frequencies, nested sets, visual representation, etc.) improving Bayesian performance have triggered some psychological debates on the underlying cognitive processes at play (for a recent analysis see Brase and Hill, 2015). Baratgin (2009) argues that these different formats facilitating Bayesian performance actually enhance the correct representation of the situation of revision in a stable universe, called the situation of focusing (Dubois and Prade, 1992, 1997) for which only Bayes' rule applies. The standard formulation of MHP prompts participants to form different representations of the situation of revision. However, when participants perceive the situation of focusing (for instance in a disambiguated version of MHP as in Baratgin and Politzer, 2010), they produce the Bayesian answer. Hence, participants cannot be considered as incoherent but only prone to an error induced by experimenters' presentation (Baratgin, 2009; Baratgin and Politzer, 2010).

$M H P$ is also used as an argument against the notion of single-case probabilities. Moser and Mulder (1994) argued that there existed two opposite rational solutions: "sticking" for a MHP proposed as a one-shot problem and "switching" for a MHP cast in a frequentist context (i.e., when imagining a sufficiently large number of games). Horgan (1995) opposed this view making explicit the correct solution for the one shot MHP and showing that switching is the only correct solution to 
both formulations. Baumann $(2005,2008)$ produced a new argument based on a generalization of MHP: the Monty Hall Problem with two players $\left(\mathrm{MHP}_{2}\right.$, see Table 1). In his view, although the two players share the same initial state of knowledge, they eventually form two different probability distributions. This point of view is opposed by Levy (2007) and by Sprenger (2010) who rightly argue that the two players do not necessarily share the same state of knowledge throughout the game in particular when their original choices differ. However, these authors do not explain the rationale of Baumann's mistake and do not explicitly define the causal structure of $M H P_{2}{ }^{1}$.

This paper will address these questions. First, the solution to $M H P_{2}$ proposed as a one shot and its causal structure will be detailed. Then, explanations for the failure of researchers investigating $\mathrm{MHP}_{2}$ will be advanced and related to the "bias" that conducts psychologists to wrongly conclude that participants' responses to $M H P$ are of a non-Bayesian nature, that is, the neglect of the Bayesian standpoint (de Finetti, 1974).

\section{Solving the Monty Hall Problem with Two Players}

Let's consider the following variables that define the properties of the possible doors $\left(D_{1}, D_{2}, D_{3}\right)$ in $M H P_{2}$ : The three variables $C$ (The host's original choice of the door in which to place the car), $Y$ (Your original choice of door) and B (Player B's original choice of door). $C, Y$, and $B$ can take any of the three values $D_{i}$ (with $i \in\{1,2,3\}$ ), respectively noted from now on $c_{i}, y_{i}$, and $b_{i}$. The variable $H$ (the host's choice when opening a door) is composed of the two complementary sub variables ' $G$ ' (the host's revealing a goat) and ' $C$ ' (the host's revealing a car). The sub variables ' $G$ ' and ' $C$ ' can take the three values $D_{i}$ (with $i \in\{1,2,3\}$ ), respectively noted from now on ' $g_{i}$ ' and ' $c_{i}$ '?

Following Walliser and Zwirn (2011), your beliefs before learning message ' $g_{3}$ ' assuming your initial choice is $D 1$ (Stage 2) can be represented as a hierarchical dynamic probabilistic structure (see Figure 1). The layer 0 depicts the four possible strategies of the host, i.e., showing a goat behind $D_{2}$ or $D_{3}$ (' $g_{2}$ ' or ' $g_{3}$ ') or showing a car when the two players have originally chosen two different doors with goats behind (' $c_{2}$ ' or ' $c_{3}$ '). Layer 1 corresponds to the three possible original choices of player $\mathrm{B}\left(b_{1}\right.$, $b_{2}$ or $\left.b_{3}\right)$. Layer 2 represents the original car placement choice of the host $\left(c_{1}, c_{2}\right.$, or $\left.c_{3}\right)$. Layer 3 is your original choice $\left(y_{1}\right)$. The probability distributions of the variables at the different layers are defined by the statement of $M H P_{2}$ with implicit and explicit hypotheses about the host's action and the players' preferences.

At Stage 4 you learn that the host will open a door with a goat behind. You know that (i) this door is either door $D_{2}$ or $D_{3}$ and (ii) the car is either behind your door $D_{1}$ or player $B$ 's originally

\footnotetext{
${ }^{1}$ The term "causal" is missing in Baumann (2005). We find Horgan's terminology of "causal structure" in Levy (2007) with the vague definition of: "the set of conditions that ultimately explains why sticking and switching have the probabilities that they do" (Levy, 2007, p. 146). Finally, Sprenger (2010, p. 337) admits that "the place of causality in the 'causal structure' of a Monty Hall game remains obscure."

${ }^{2}$ We use here quotes for all sub-variables related to the host's actions during the game.
}

chosen door. Hence you focus on the subset where ' $g_{2}$ ' or ' $g_{3}$ ' is true (the continuous lines in Figure 1). You are better off sticking to your initial choice $D_{1}$.

$$
P\left(c_{1} \mid y_{1}{ }^{\prime} G^{\prime}\right)=3 / 7>2 / 7=P\left(c_{2} \mid y_{1}^{\prime} G^{\prime}\right)=P\left(c_{3} \mid y_{1}{ }^{\prime} G^{\prime}\right)
$$

Second at Stage 5 the host opens door $D_{3}$ and reveals a goat behind. You focus on the subset where ' $g_{3}$ ' is true (the bold lines in Figure 1). This information combined with your original choice of door provides information about the door behind which Monty placed the car. You are better off switching to door $D_{2}$.

$$
P\left(c_{1} \mid y_{1}{ }^{\prime} g_{3}^{\prime}\right)=3 / 7<4 / 7=P\left(c_{2} \mid y_{1}{ }^{\prime} g_{3}{ }^{\prime}\right)
$$

Finally at Stage 6 you learn what was player B's original choice. On the one hand, it can coincide with yours $\left(b_{1}\right)$. Both players are then exactly in the same situation with the same common knowledge. $M H P_{2}$ amounts to $M H P$. Hence, you know that $C$ is twice as likely to have the value $c_{2}$ as to have the value $c_{1}$. The best strategy is to switch from your original choice to the other closed door $D_{2}$.

$$
P\left(c_{1} \mid y_{1} b_{1}{ }^{\prime} g_{3}{ }^{\prime}\right)=1 / 3<2 / 3=P\left(c_{2} \mid y_{1} b_{1}{ }^{\prime} g_{3}{ }^{\prime}\right)
$$

On the other hand you may learn that player $B$ 's original choice is different from yours $\left(b_{2}\right)$. In this case there is no best strategy and you are indifferent to sticking or switching.

$$
P\left(c_{1} \mid y_{1} b_{2}{ }^{\prime} g_{3}{ }^{\prime}\right)=1 / 2=P\left(c_{2} \mid y_{1} b_{2}{ }^{\prime} g_{3}{ }^{\prime}\right)
$$

\section{The Collider Principle}

Glymour (2001) was the first to identify the causal structure in $M H P$ as a situation where two independent variables that mutually influence another variable are dependent conditional on the value of the variable they both affect. In $M \mathrm{HP}_{2}$, the three independent variables $Y, B$, and $C$ symmetrically influencing (colliding with) another variable $H$ (common effect) actually appear dependent conditionally on the values of the variable $H$. Hence observing the value of $H$ provides some information on the possible values of $Y, B$ or $C$. In the same way, knowing the values of any couple of variables $(C, H),(B, H)$, and $(Y, H)$ provides some information about the values of couples $(Y, B),(Y$, $C)$, and $(B, C)$, respectively. Finally observing the values of triples ( $Y, C, H),(B, C, H),(Y, B, H)$, respectively determines the values of variables $B, Y$, and $C$. Solving $M H P_{2}$ as a one shot game relies on the latter triple $(Y, B, H)$. It is easy when two variables are fixed to derive some qualitative predictions (Wellman and Henrion, 1993). For instance, $M H P_{2}$ 's solution supports a phenomenon of reversal decision resulting from this collider principle. On learning $H={ }^{\prime} g_{3}$ ' given your original choice $\left(Y=y_{1}\right)$ the likelihoods that $B$ and $C$ equal $b_{2}$ and $c_{2}$, respectively, are higher than the likelihoods that $B$ and $C$ equal $b_{1}$ and $c_{1}$, respectively. However, if in addition you learn that $B$ equals $b_{1}$ then the outcome $c_{2}$ seems the more likely. However, if you learn that $B$ equals $b_{2}$ then the probabilities for the car being behind either $D_{1}$ or $D_{2}$ are even. 
TABLE 1 | The six sequential stages of $M H P_{2}$.

\begin{tabular}{|c|c|}
\hline Stages & Descriptions \\
\hline
\end{tabular}

Stage 2 Each player picks a door and neither player is informed of the other player's choice. Let's assume for the sake of convenience that you are player $A$ and you initially select door $D_{1}$.

Stage 3 The host, who knows where the car is, tells you: "In the case where player $B$ has chosen the same door as you (here $D_{1}$ ), I will show you one door (out of the two other doors) behind which there is a goat." It is assumed that both players know that the host has no preference between the two remaining doors $\left(D_{2}\right.$ and $D_{3}$ ) to show a goat should the car be behind $D_{1}$. Then the host continues: "In the case where player $B$ has picked another door, I will always open the third door -chosen by neither player- even if the car is behind it." In this latter case when the host reveals a car, both players (you and player $B$ ) win and have no decision to make; the game stops.

Stage 4 The host says "I will open a door to reveal a goat" and then asks both players still ignorant of the other player's original choice: "To win the car should you stick to your original choice or switch to another door (as far as you are concerned door $D_{2}$ or door $D_{3}$ )."

Stage 5 The host opens a door (for example $D_{3}$ ), reveals a goat and then asks both players again: "To win the car, should you stick to your original choice or switch to the other closed door (door $D_{2}$ in your case)?"

Stage 6 Each player reveals her or his original choice and must then decide knowing the other player's choice whether to stick to her/his door $\left(D_{1}\right.$ in your case) or to switch door $\left(D_{2} \text { in your case }\right)^{b}$.

aln the case where both players succeed in their door choice with the car, they each get a car. Hence, as noted by Sprenger (2010), there is no real competition between both players.

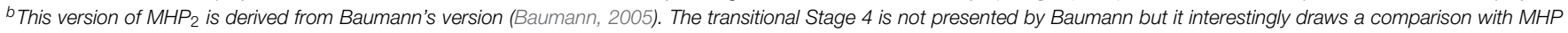
where this information is not informative. We also added the Stage 6 to find again MHP in the situation where the two players have originally chosen the same door.

Layer 3

(your initial choice, $y_{1}$ )

Layer 2

(doors property, $C$ )

Layer 1

(B's choice, $B$ )

Layer 0 (host's opening, $H$ )

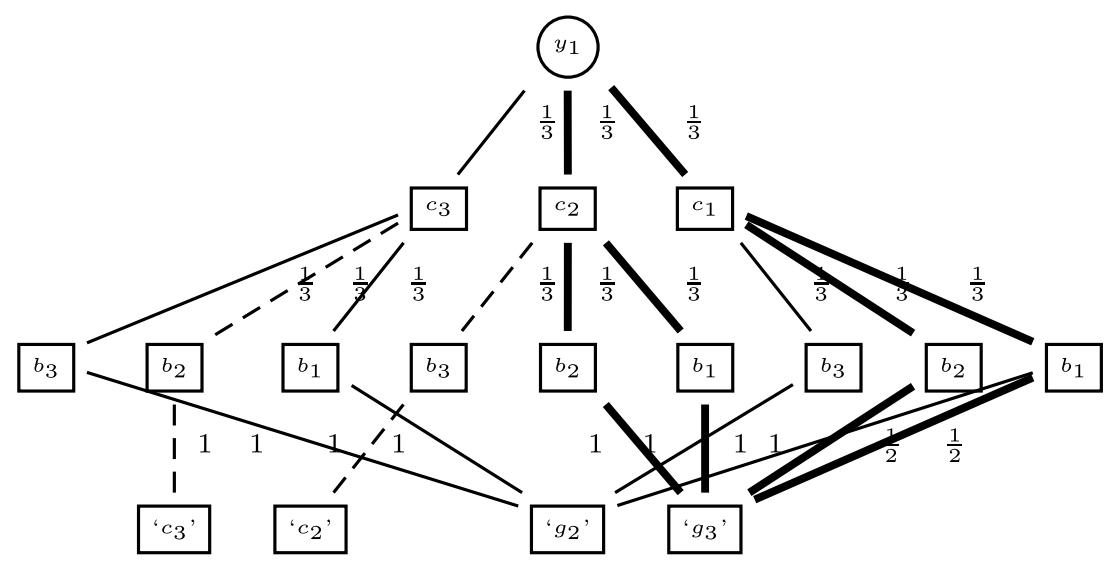

FIGURE 1 | The general tri-probabilistic structure of $M \mathrm{HP}_{2}$ before learning message ' $g_{3}$ ' assuming your initial choice is $D_{1}\left(Y=y_{1}\right)$. The continuous lines correspond to the subset left after compiling information at
Stage 4 and the bold lines to the subset left after compiling the information at Stage 5. Conversely the dashed lines represent the initial structure dropped out at Stage 4.
Recent studies have provided some evidence that "naive" adults and also children make correct qualitative predictions in collider principle situations when pairs of causal conditionals are explicitly presented (Ali et al., 2010, 2011). Precisely in $M H P$, participants perform better when the relation between the player's original choice and the host's strategy is explicit in conditional form (Macchi and Girotto, 1994, cited in JohnsonLaird et al., 1999). In the same way, when participants can construct a representation analogous to Figure 1 for $M H P$ using a graph or by means of physical handling, participants' performance improves significantly (Yamagishi, 2003; Baratgin and Politzer, 2010). Thus, it seems that when participants can infer the causal structure of $M H P$ by physical or explanatory cues, they are able to solve MHP (Burns and Wieth, 2004; Chater and Oaksford, 2006).

\section{The Neglect of the Bayesian Standpoint}

De Finetti's subjective Bayesian standpoint proposes that individuals form two levels of knowledge (de Finetti, 1980; Baratgin and Politzer, in press): 
- An elementary level of knowledge of an event $E$ that is always conditioned on an individual's specific state of knowledge $\left\{H_{0}\right\}$ at this time. Furthermore, any event is actually a tri-event (the third value representing ignorance between true event and false event).

- A meta-level of knowledge concerning the degrees of belief of an individual. Here ignorance is specified, and refined, into degrees of belief. From an inferential point of view, your subjective probability of this event $E$ at time $t_{0}$ is always conditional on your current state of knowledge $\left\{H_{0}\right\}$ [and should be written $P\left(E \mid H_{0}\right)$ ]. It is coherent if (i) it follows the axiom of additive probabilities ${ }^{3}$ and (ii) when acquiring a new knowledge $H$, your probability also depends on this new knowledge $\left\{H_{0} H\right\}$ [and should be written $\left.P\left(E \mid H_{0} H\right)\right]$.

A person dismissing the Bayesian standpoint considers the probability of a single event as questionable as compared to a "frequentist" conception of probability. She takes the frequentist conception to be the "correct" comparative representation, and confines Bayesianism to just a set of Bayesian techniques (de Finetti, 1974). In the psychological literature this "bias" leads to two significant mistakes: (i) to the neglect of pragmatic constraints on the methodology (to understand $H_{0}$ and $H$ ); (ii) to the conclusion that people's behavior is "non-Bayesian," even when the behavior does not violate Bayesian coherence (Baratgin, 2002; Mandel, 2014a). In the analysis of $M H P_{2}$, this bias is characterized by inadequate terminology and interpretation of the revision situation.

\subsection{The Use of an "Ambiguous Terminology"}

For a subjective Bayesian, an event $E$ always refers to a certain outcome in a single well-defined case (a unit in which the definition is unambiguous and complete) and cannot be used in a generic sense (such as a collection of "identical events"). There is no repetition of the same event but a succession of many distinct events, which can be different illustrations of the same phenomenon. In Moser and Mulder (1994), Baumann (2005), Levy (2007), and Baumann (2008), $M H P_{2}$ is presented in an ambiguously termed way (de Finetti, 1977/1981, p. 357). The variables are considered as trials of the same phenomenon without completely specifying them and their possible values. Every specific door corresponds to a generic door $D$ that is characterized by two properties: having a car $(C)$ or a goat $(G)$ behind it. Every player's original door choice is analyzed by its correspondence with $C$ and $G$. The host's door opening ' $H$ ' is characterized by the two sub-classes ' $G$ ' and ' $C$ '. The players' final decisions to win the car are commingled and considered to pertain to the same classes of events "to stick," "to switch" or "nothing."

Following this frequentist "jargon" (de Finetti, 1979a,b), $\mathrm{MHP}_{2}$ is analyzed as an observation of a repetitive problem where the different variables are interchangeable in function of the host's car placement. Instead of considering each player with specific states of knowledge relative to each stage of $\mathrm{MHP}_{2}$

${ }^{3}$ See for example on this special research topic (Cruz et al., 2015; Evans et al., 2015; Mandel, 2015) and also (Politzer and Baratgin, in press). both players are assumed to have a common knowledge at each stage of the game. Their probabilities that there is a car behind one of the two remaining doors (after the door with a goat behind was opened) is $3 / 7$ for the door originally chosen and $4 / 7$ for the other door. Thus, imagining they made a different original choice, each door can be associated with two different probabilities (3/7 and 4/7) illustrating Bauman's paradox. Now, if we consider the specific knowledge of each player, the paradox disappears. In Stages 4 and 5, player B's probabilities on $c_{1}$ and $c_{2}$ are identical to your probabilities (relations 1-3) when his/her specific initial state knowledge is identical to yours (his/her original choice is $b_{1}$ ). Conversely when his/her original choice is $b_{2}$, his/her state of knowledge is different from yours and his/her probabilities correspond to different probabilities (relations 5 and 6):

$$
\begin{gathered}
P\left(c_{1} \mid b_{2}{ }^{\prime} G^{\prime}\right)=P\left(c_{3} \mid b_{2}{ }^{\prime} G^{\prime}\right)=2 / 7<3 / 7=P\left(c_{2} \mid b_{2}{ }^{`} G^{\prime}\right) \\
P\left(c_{1} \mid b_{2}{ }^{\prime} g_{3}{ }^{\prime}\right)=4 / 7>3 / 7=P\left(c_{2} \mid b_{2}{ }^{\prime} g_{3}{ }^{\prime}\right)
\end{gathered}
$$

However, player B's decisions are identical: sticking at Stage 4 and switching at Stage 5. At Stage 6, both players have an identical state of knowledge and probabilities (relation 4).

\subsection{Neglect of the Situation of Focusing}

$M H P_{2}$ illustrates that the situation of revision implied by the Bayesian standpoint is a process of focusing on a subset of the initial state of knowledge $\left\{H_{0}\right\}$ (de Finetti, 1957; Dubois and Prade, 1992, 1997). It is assumed that one object is selected from the universe and that a message releases information about it. A reference class different from the initial one is consequently considered by focusing attention on a given subset of the original set that complies with the information about the selected object. This is not a temporal revision process because the information ' $g_{3}$ ' just focuses on the selection of a particular posterior probability that was virtually available (among others) (see the bold lines of Figure 1). Yet participants in MHP seem to adopt (for pragmatic reasons) another representation of the revision situation, known as updating (Katsuno and Mendelzon, 1992; Walliser and Zwirn, 2002) in which, they infer from the message ' $g_{3}$ ' the information as "door $D_{3}$ have been removed," and conceive a new probability distribution consistent with this new problem (Baratgin and Politzer, 2007, 2010; Baratgin, 2009). In this representation there is obviously no collider effect because, in this new problem with two doors, the variables $Y$ and $H$ always remain independent after the information is provided by the host. Participants form a new distribution of probability $P^{\prime}$ for this new game ${ }^{4}$. Two typical analyses are consistent with this interpretation:

The stick or switch response: if you originally chose door $D_{1}$ and the host opens door $D_{3}$ with a goat behind, the worlds $c_{1}$ and $c_{2}$ are evenly close (in fact proportionally to their prior probabilities) to the invalidated world $c_{3}$. The weight of $c_{3}$

${ }^{4} P^{\prime}$ along the following process: (i) The worlds ' $c_{3}$ ' and ' $g_{2}$ ' are canceled and a simpler probabilistic structure composed of the two worlds $\left(c_{1}, c_{2}\right)$ is obtained, (ii) The new distribution $P^{\prime}$ stems from a redistribution of the weights (the probabilities) of the removed worlds on the two remaining worlds. 
is redistributed proportionally on $c_{1}$ and $c_{2}$. This is MHP's solution in the updating context proposed by Dubois and Prade (1992).

$$
\begin{aligned}
P^{\prime}\left(c_{1} \mid y_{1}\right) & =P\left(c_{1} \mid y_{1}\right)+1 / 2 P\left(c_{3} \mid y_{1}\right)=1 / 2 \\
& =P\left(c_{2} \mid y_{1}\right)+1 / 2 P\left(c_{3} \mid y_{1}\right)=P^{\prime}\left(c_{2} \mid y_{1}\right)
\end{aligned}
$$

It corresponds to the "equiprobability" solution given by nearly all participants to $M H P$ but also by some experts in their analysis of $M H P$ in a single isolated situation (Moser and Mulder, 1994) and of $\mathrm{MHP}_{2}$ (Levy, 2007).

The switch response: The worlds $c_{3}$ and $c_{2}$ (the two doors not originally chosen by the player) are considered closer. The probability of the invalidated world $c_{3}$ is transferred to $c_{2}$ alone. This is MHP's solution in the updating context proposed by Cross (2000).

$$
\begin{aligned}
& P^{\prime}\left(c_{1} \mid y_{1}\right)=P\left(c_{1} \mid y_{1}\right)=1 / 3 \text { and } \\
& P^{\prime}\left(c_{2} \mid y_{1}\right)=P\left(c_{2} \mid y_{1}\right)+P\left(c_{3} \mid y_{1}\right)=2 / 3
\end{aligned}
$$

This response is given by only few participants to MHP (see for review Baratgin, 2009). It corresponds to Moser and Mulder's explanation for MHP's solution in a suitable long run of relevantly similar situations. To explain the "causal structure" of MHP, Levy (2007) proposed also a process in line with this updating interpretation. However, it is difficult here to support the "switch" response to $M H P_{2}$ with the symmetric

\section{References}

Ali, N., Chater, N., and Oaksford, M. (2011). The mental representation of causal conditional reasoning: mental models or causal models. Cognition 119, 403-418. doi: 10.1016/j.cognition.2011.02.005

Ali, N., Schlottmann, A., Shaw, A., Chater, N., and Oaksford, M. (2010). "Causal discounting and conditional reasoning in children," in Cognition and Conditionals. Probability and Logic in Human Thinking, eds M. Oaksford and N. Chater (New York, NY: Oxford University Press), $117-134$.

Baratgin, J. (2002). Is the human mind definitely not bayesian? A review of the various arguments. Curr. Psychol. Cogn. 21, 653-682.

Baratgin, J. (2009). Updating our beliefs about inconsistency: the Monty-Hall case. Math. Soc. Sci. 57, 67-95. doi: 10.1016/j.mathsocsci.2008.08.006

Baratgin, J., and Politzer, G. (2007). The psychology of dynamic probability judgment: order effect, normative theories and experimental methodology. Mind Soc. 6, 53-66. doi: 10.1007/s11299-006-0025-z

Baratgin, J., and Politzer, G. (2010). Updating: a psychologically basic situation of probability revision. Think. Reason. 16, 253-287. doi: 10.1080/13546783.2010.519564

Baratgin, J., and Politzer, G. (in press). "Logic, probability and inference: a methodology for a new paradigm," in Cognitive Unconscious and Human Rationality, eds L. Macchi, M. Bagassi, and R. Viale (Cambridge, MA: MIT Press).

Baratgin, J., and Walliser, B. (2010). Sleeping beauty and the absent-minded driver. Theory Decis. 69, 489-496. doi: 10.1007/s11238-010-9215-6

Baumann, P. (2005). Three doors, two players, and single-case probabilities. Am. Philos. Q. 42, 71-79. Available online at: http://www.jstor.org/stable/20010183? seq=1\#page_scan_tab_contents

Baumann, P. (2008). Single-case probabilities and the case of Monty Hall: Levy's view. Synthese 162, 265-273. doi: 10.1007/s11229-007-9185-6 role of the two players (Levy, 2007). Thus, the "stick or switch response" should be privileged to solve $M H P_{2}$ in an updating representation.

\section{Conclusion}

This paper describes the supposedly paradoxical solutions attributed to $M H P_{2}$ from the perspective of a thorough Bayesian standpoint perspective. It outlines the methodological care that one should take to comprehend the problem in relation to the single case terminology and the focusing context of revision. Not taking into account these features prevents one from fully grasping the probabilistic temporal dynamics of the problem and consequently the corresponding causal collider structure.

Psychologists who study subjective Bayesian reasoning should carefully formulate the statement without ambiguity and respect the Bayesian standpoint. This is also true especially for complex problems (such as the Sleeping Beauty problem Baratgin and Walliser, 2010; Mandel, 2014b) in which different solutions can be envisaged depending on the interpretations made by participants.

\section{Acknowledgments}

Financial support for this work was provided by a grant from the ANR Chorus 2011 (project BTAFDOC). The author thanks N. Cruz, G. Politzer, and B. Walliser for very helpful comments on a previous draft of this manuscript.

Brase, G. L., and Hill, W. T. (2015). Good fences make for good neighbors but bad science: a review of what improves bayesian reasoning and why. Front. Psychol. 6:340. doi: 10.3389/fpsyg.2015.00340

Burns, B., and Wieth, M. (2004). The collider principle in causal reasoning: why the Monty Hall dilemma is so hard. J. Exp. Psychol. 133, 434-449. doi: 10.1037/0096-3445.133.3.434

Chater, N., and Oaksford, M. (2006). "Information sampling and adaptive cognition," in Mental Mechanisms. Speculations on Human Causal Learning and Reasoning, eds K. Fiedler and P. Juslin (Cambridge: Cambridge University Press), 210-236.

Cross, C. B. (2000). A characterization of imaging in terms of Popper functions. Philos. Sci. 67, 316-338. doi: 10.1086/392778

Cruz, N., Baratgin, J., Oaksford, M., and Over, D. E. (2015). Bayesian reasoning with ifs and ands and ors. Front. Psychol. 6:192. doi: 10.3389/fpsyg.2015.00192

de Finetti, B. (1957). L'informazione, il ragionamento, l'inconscio nei rapporti con la previsione. Lindustria 2, 3-27.

de Finetti, B. (1974). Bayesianism: its unifying role for both the foundations and applications of statistics. Int. Stat. Rev. 42, 117-130.

de Finetti, B. (1977/1981). La probabilità: guardarsi dalle contraffazioni. Scientia 111, 255-281.

de Finetti, B. (1979a). Jargon-derived and underlying ambiguity in the field of probability. Scientia 114, 713-716.

de Finetti, B. (1979b). Probability and exchangeability from a subjective point of view. Int. Stat. Rev. 47, 129-135.

de Finetti, B. (1980). "Voice probabilitá," in Encyclopedia, (Torino: Einaudi), $1146-1187$.

Dubois, D., and Prade, H. (1992). Evidence, knowledge, and belief functions. Int. J. Approx. Reason. 6, 295-319. doi: 10.1016/0888-613X(92)90027-W

Dubois, D., and Prade, H. (1997). "Focusing vs. belief revision: a fundamental distinction when dealing with generic knowledge," in Qualitative and Quantitative Practical Reasoning, Vol. 1244 of Lecture Notes in Computer 
Science, eds D. Gabbay, R. Kruse, A. Nonnengart, and H. Ohlbach (Berlin; Heidelberg: Springer), 96-107.

Evans, J. S. Thompson, V. A., and Over, D. E. (2015). Uncertain deduction and conditional reasoning. Front. Psychol. 6:398. doi: 10.3389/fpsyg.2015.00398

Glymour, C. (2001). The Mind's Arrows: Bayes Nets and Graphical Causal Models in Psychology. Cambridge, MA: The MIT Press.

Horgan, T. (1995). Let's make a deal. Philos. Pap. 24, 209-222. doi: 10.1080/05568649509506532

Johnson-Laird P. N., Legrenzi, P., Girotto, V., and Sonino-Legrenzi, M. S. (1999). Naive probability: a mental model theory of extensional reasoning. Psychol. Rev. 106, 62-88. doi: 10.1037/0033-295X.106.1.62

Katsuno, A., and Mendelzon, A. (1992). "On the difference between updating a knowledge base and revising it," in Belief Revision, ed P. Gärdenfors (Cambridge: Cambridge University Press), 183-203.

Levy, K. (2007). Baumann on the Monty Hall problem and single-case probabilities. Synthese 158, 139-151. doi: 10.1007/s11229-006-9065-5

Macchi, L., and Girotto, V. (1994). "Probabilistic reasoning with conditional probabilities: the three boxes paradox," in Paper presented at the Annual Meeting of the Society for Judgement and Decision Making. (St. Louis, MO)

Mandel, D. R. (2014a). The psychology of bayesian reasoning. Front. Psychol. 5:1144. doi: 10.3389/fpsyg.2014.01144

Mandel, D. R. (2014b). Visual representation of rational belief revision: another look at the sleeping beauty problem. Front. Psychol. 5:1232. doi: 10.3389/fpsyg.2014.01232

Mandel, D. R. (2015). Instruction in information structuring improves bayesian judgment in intelligence analysts. Front. Psychol. 6:387. doi: 10.3389/fpsyg.2015.00387

Moser, P. K., and Mulder, D. H. (1994). Probability in rational decision-making. Philos. Pap. 23, 109-128. doi: 10.1080/05568649409506416
Politzer, G., and Baratgin, J. (in press). Deductive schemas with uncertain premises using qualitative probability expressions. Think. Reason. doi: 10.1080/13546783.2015.1052561

Sprenger, J. (2010). Probability, rational single-case decisions and the Monty Hall problem. Synthese 174, 331-340. doi: 10.1007/s11229-008-9455-y

Tubau, E., Aguilar-Lleyda, D., and Johnson, E. D. (2015). Reasoning and choice in the monty hall dilemma (mhd): implications for improving bayesian reasoning. Front. Psychol. 6:353. doi: 10.3389/fpsyg.2015.00353

Walliser, B., and Zwirn, D. (2002). Can Bayes rule be justified by cognitive rationality principles? Theory Decis. 53, 95-135. doi: 10.1023/A:10212271 06744

Walliser, B., and Zwirn, D. (2011). Change rules for hierarchical beliefs. Int. J. Approx. Reason. 52, 166-183. doi: 10.1016/j.ijar.2009.11.005

Wellman, M., and Henrion, M. (1993). Explaining 'explaining away'. IEEE Trans. Pattern Anal. Mach. Intell. 15, 287-292.

Yamagishi, K. (2003). Facilitating normative judgments of conditional probability: frequency or nested sets? Exp. Psychol. 50, 97-106. doi: 10.1026/16183169.50.2.97

Conflict of Interest Statement: The author declares that the research was conducted in the absence of any commercial or financial relationships that could be construed as a potential conflict of interest.

Copyright $\odot 2015$ Baratgin. This is an open-access article distributed under the terms of the Creative Commons Attribution License (CC BY). The use, distribution or reproduction in other forums is permitted, provided the original author(s) or licensor are credited and that the original publication in this journal is cited, in accordance with accepted academic practice. No use, distribution or reproduction is permitted which does not comply with these terms. 